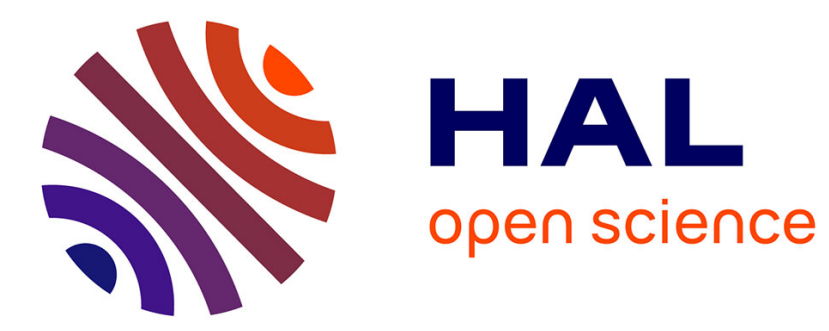

\title{
The search for exotic nuclei and the new heavy ion machines
}

\author{
C. Detraz
}

\section{To cite this version:}

C. Detraz. The search for exotic nuclei and the new heavy ion machines. Nuclear Physics A, 1983, 409, pp.353c-360c. 10.1016/0375-9474(83)90697-8 . in2p3-00258287

\section{HAL Id: in2p3-00258287 https://hal.in2p3.fr/in2p3-00258287}

Submitted on 25 Jun 2020

HAL is a multi-disciplinary open access archive for the deposit and dissemination of scientific research documents, whether they are published or not. The documents may come from teaching and research institutions in France or abroad, or from public or private research centers.
L'archive ouverte pluridisciplinaire HAL, est destinée au dépôt et à la diffusion de documents scientifiques de niveau recherche, publiés ou non, émanant des établissements d'enseignement et de recherche français ou étrangers, des laboratoires publics ou privés. 


\title{
THE SEARCH FOR EXOTIC NUCLEI AND THE NEW HEAVY ION MACHINES
}

\section{Claude DETRAZ}

\author{
GANIL, B.P. 5027, 14021 CAEN Cédex \\ France
}

\begin{abstract}
The possibilities opened by the new heavy-ion accelerators, with energies up to $100 \mathrm{MeV}$ per nucleon, for the study of nuclei far from stability, are discussed.
\end{abstract}

\section{Introduction}

The limits of our knowledge of nuclei keep extending further and further away from stability. This moving frontier is getting closer to the proton and neutron drip lines. The challenge is to extend the doma in of observed isotopes and to collect quantitative information on their properties. An overview of the field is provided by the proceedings of the last of the periodical conferences on nuclei far from the valley of $\beta$ stability ${ }^{1}$ ).

This paper only deals with the following limited question. Does the availability of new heavy ion accelerators provide new ways to extend the doma in of observed nuclei. The new accelerators of interest are those which bring an increase of energy above the classical Tandem or cyclotron range, i.e. which reach 10 to $100 \mathrm{MeV}$ for nucleon, such as GANIL, SARA, the MSU cryogenic cyclotrons, the RIKEN and Catania projects, etc...

In the energy range considered, two important methods used so far to produce exotic nuclei are going to be of lesser importance because their cross sections decrease rapidly with increasing heavy ion energy. The first one uses deep inelastic reactions, which allowed a remarkable breakthrough in the sixties with the pioneering work of Volkov ${ }^{2}$ ) and has been extensively used since then, as exemplified at the Helsingor conference ${ }^{1}$ ). The second one is compound nucleus formation, especially useful to produce neutron deficient nuclei, since it best operates at an energy close to the Coulomb barrier, as shown by the recent discovery of elements 107 and $109^{3}$ ).

But other processes deserve special attention: two-body reactions and fragmentation processes.

\section{Study of exotic nuclei by two-body reactions}

The observation of a $A+B \rightarrow C+D$ ground state to ground state reaction through the measurement of the energy of the $C$ or $D$ nucleus at a given angle provides knowledge of the reaction Q-value. If three of the four nuclear masses are known the fourth one is readily deduced. This method also allows for the observation of excited states of the $C$ and $D$ nuclei.

These reactions induced by light ions such as ${ }^{3} \mathrm{He}$ were extensively used in the sixties to measure the mass of neutron deficient isotopes by e.g. $\left({ }^{3} \mathrm{He},{ }^{6} \mathrm{He}\right)$ reactions ${ }^{4}$ ). A systematics of reaction cross sections for these exotic transfers was reasonably established ${ }^{5}$ ). The occurrence of heavy ion beams extended the possibilities of this method (see ref ${ }^{2}$ for typical examples) in the seventies. For instance, two-proton transfer reactions have proved remarkably fruitfui ${ }^{6-7}$ ). Improvements in the detection techniques and beam performances have allowed the measurement of cross sections down to very small 1 imits corresponding to a few events per day. It seems unlikely that at Tandem energies this method could bring many more results. Thus the question arises whether the increase in projectile energies 
allowed by the new accelerators might induce a significant increase in cross sections for exotic transfer reactions. The answer cannot come from systematics alone because of the observed large dispersion between $d \sigma / d \Omega$ values of apparently situilar reactions.

A general argument can be made which suggests that an optimum incident eneray exists for exotic reactions with large negative Q-values.

At low energies, say 50 to $100 \mathrm{MeV}$ for $\mathrm{C}$ or 0 beams, the cross sections are hindered by severe mismatch effects. This can be argued in two ways : either by saying that large Q-values correspond to large kinetic energy changes between the initial and final channels which can be matched only by large angular momentum transfers, a condition hardly compatible with most ground state transitions; or by invoking the Q-window open by matching the Coulomb trajectories in the initial and final channels, which is not usually that far from $0 \mathrm{MeV}$ to encompass the extremely negative $Q$-values of the exotic transfer reactions cf interest.

Further, there are some experimental indications that an increase in energy moves the cross section. Such is the case for the $\left({ }^{18} 0,{ }^{2.7} \mathrm{C}\right),\left({ }^{18} 0,{ }^{18} \mathrm{C}\right)$, $(0,1 E)$ reactions where factors around $10^{9-10}, 3^{11-12}$ and $10^{13-14}$, respective$1 y$, are gained when the incident energy rises from around $95 \mathrm{MeV}$ to $110 \mathrm{MeV}$.

On the other hand, at very high energies, the relative velocity of the two interacting nuclei is so high that it strongly hinders the smooth velocitytatched transfer of niscleons from orbits in one nucleus onto orbits in the other.

This one might expect intermediate energies, at some tens of MeV per nucleon, to correspond to an optimum cross section.

A quantitative guide line to tackle that problem can be found in the semiclessical descriptions of heavy ion discussion. In particular F. Naulin ${ }^{14}$ )applies the kinematical matching conditions derived by $D . M .\left(B r_{i n k}{ }^{15}\right)$. These require the conservation in a one-step transfer of the transferred nucleon wave number along the beam axis, $\Delta k=0$, and of the total angular momentum, $\Delta L=0$. Actually quantal effects make for less stringent conditions, $\Delta k$ and $\Delta L$ being only smaller than reference values $\Delta k_{0}$ and $\Delta L_{0}$ linked to uncertainties on the transfer location and the binding energy, respectively ${ }^{15}$ ). (It is extended by Naul in to multi-nucleon iransfer and two-step reactions such as e.g. $-2 n+p$ transfers). A hindrance torameter which can reflect the degree of mismatch in a one-step direct reaction transfer is thus defined as

$$
H=\Delta k / \Delta k_{0}+\Delta L / \Delta L_{0} .
$$

One should then observe an anti correlation between $H$ and the reaction cross section, typically measured at forward angles. From a compilation ${ }^{14}$ ) of published cross sections for two, three and four nucleon transfer the points of fig. 1 are obtained. They roughly exhibit the anti correlation, at least within one order of a agnitude, as expected since no provision is made for nuclear structure effects. The variation of $H$ can thus be used to predict gross variations of the cross sec-

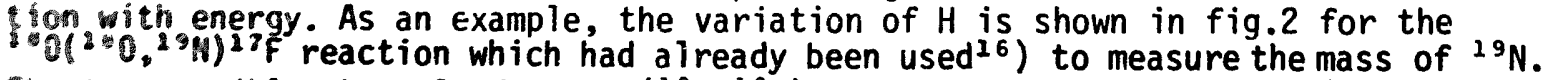
The two possible channels for the $\left({ }^{18} 0,{ }^{19} \mathrm{~N}\right)$ reaction, namely $-p+2 n$ or $2 n-p$, ore gaen into accourit. The results shown in fig. 2 indicate that a broad minimum in $H$ appaears at some 15 or $20 \mathrm{MeV}$ per nucleon for the laboratory incident eneroy. Yet the reduction in $H$, still important in the upper range of Tandem energies. is moderate afterwards.

Fron this result and similar ones ${ }^{14}$ ) it seems fair to conclude that only moderate $g^{\text {ins }}$ are to be expected for exotic two-body reactions between Tandem pergies ar. "he energy range opened by the new accelerators at a few tens of MeV

This conclusion should be qualified by one word of cautiori. The exotic reactions of interest exhaust in general less than one part in a million of the total cross section. Thus they are so marginal that dynamical treatments such as the one ${ }^{15}$ ) used above which intend to describe the first order behaviour of the reaction might be irrelevant. 


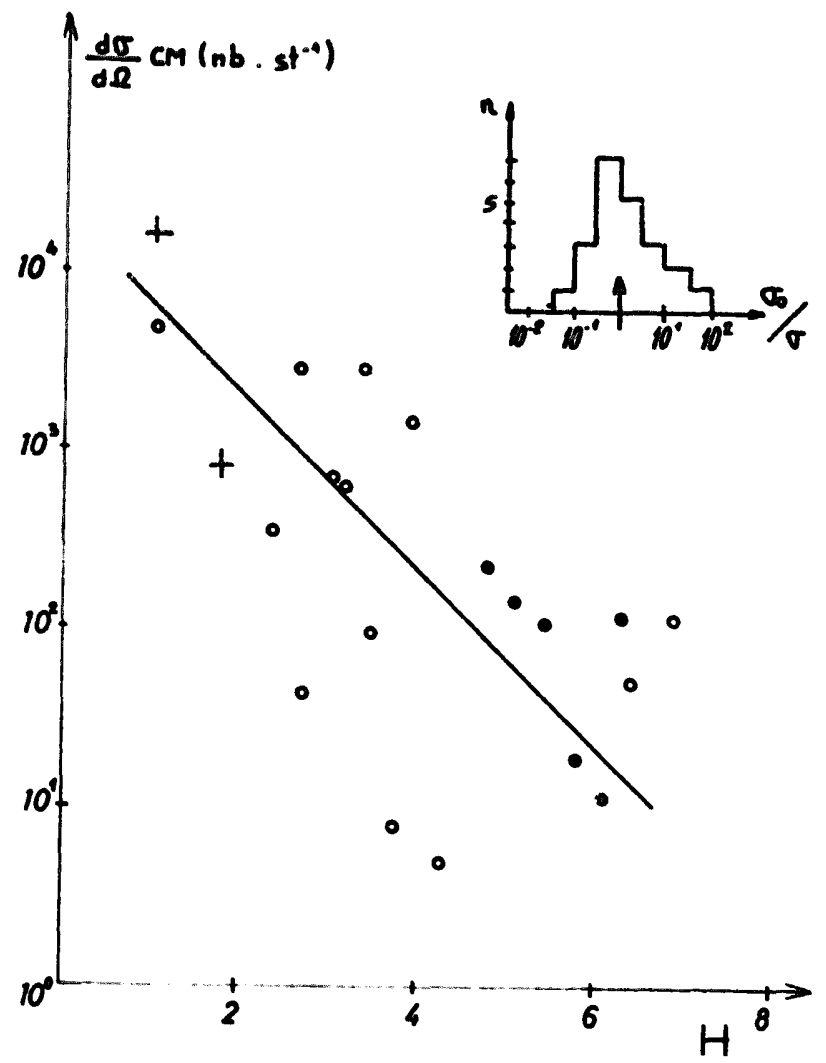

Fig. 1

Fig. 2

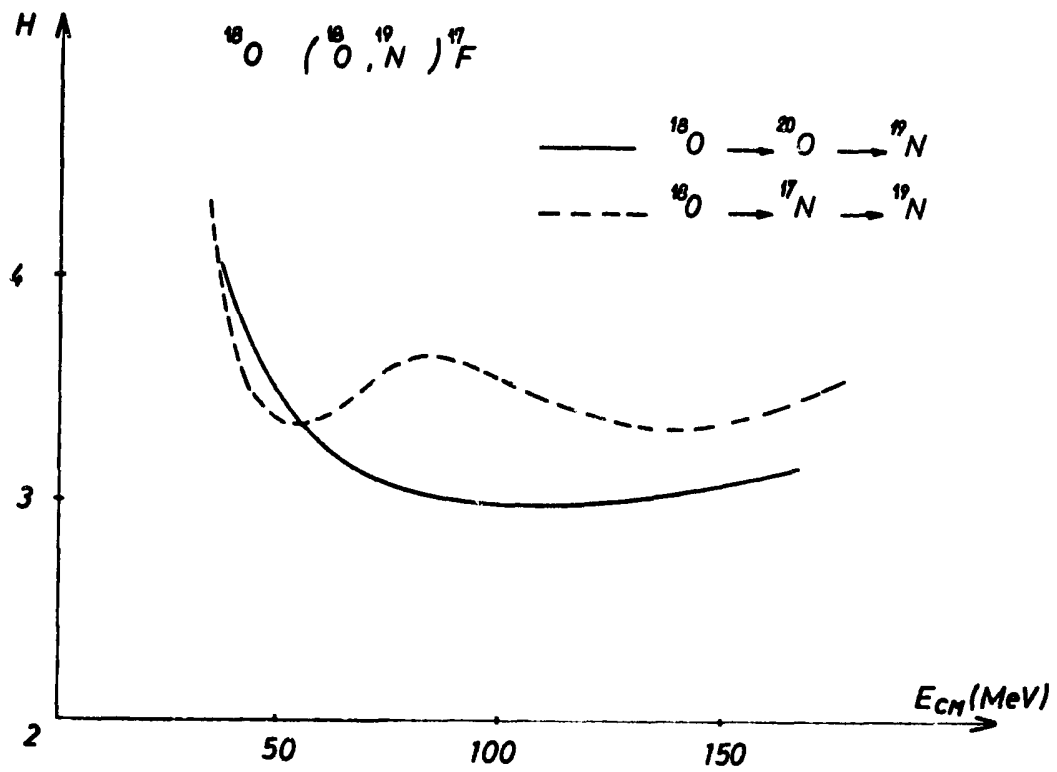

Fig. 1 A compilation, taken from $\mathrm{F}$. $\mathrm{Naul} \mathrm{in}^{14}$ ), of the experimental cross section for two $(+)$, three $(0)$ and four ( $(0)$ nucleon transfers versus the hindrance factor $H$ determined from semi-classical matching conditions as discussed in the text. The line is an empirical fit to the rough anti correlation observed, the insert shows the deviation of the experiment cross section $(\sigma)$ to the one $\left(\sigma_{0}\right)$ corresponding to the linear fit.

Fig. 2 Variation of $\mathrm{H}$, the hindrance factor discussed in the text, with the incident energy for the ${ }^{18} 0\left({ }^{18} 0,{ }^{19} \mathrm{~N}\right)^{17} \mathrm{~F}$ reaction for two possible reaction mechanism (taken from $F$. Naul in ${ }^{14}$ ). 


\section{Fragmentation processes}

The new heavy ion accelerators allow one to deposit important energies inside the target nucleus. That such a process, when induced by proton, or also $d,{ }^{3} \mathrm{H}, \alpha$, or II particles, leads to large fragmentation cross section is well documented ${ }^{7}$ ). The yield of a given $(N, Z)$ fragment is roughly isotropic and it smoothly varies with the mass of the target nucleus, the $Z$ and $N$ values of the fragment. The fragment energy exponentially decreases above the Coulomb barrier. The most striking feature of the cross section is its steep increase with incident energy up to $i$ or $2 \mathrm{GeV}$ where a plateau is reached. Similar behaviour now appears to be true also for heavy ion bombardment ${ }^{19}$ ). Therefore the new heavy ion accelerators provide new ways to deposit the few GeV's necessary for fragmentation of heavy targets to occur with large cross sections.

The heavy ion facilities will not be as effective as high energy proton accelerators as far as yields are concerned because of thinner targets and frequently lower beam intensities. However they will imply easier shielding.

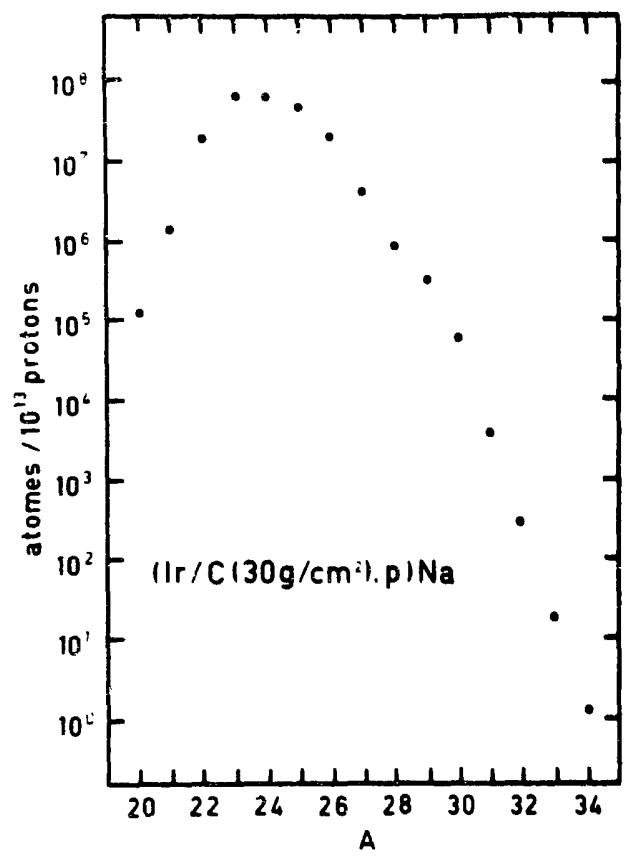

Fig. 3 Yield of $\mathrm{Na}$ isotopes analysed by on-line mass spectroscopy when produced by high-energy proton bombardment on Iridium (taken from ref ${ }^{28}$ ).

The effectiveness of high energy fragmentation is abundantly illustrated in particular by work performed at CFRN at both the proton synchrotron by the Orsay group (Fig.3), and the Isolde facility (see ref ${ }^{1}$ ). It is the feeling of this author that the fruitfulness of this process is still far from having been exhausted in the study of exotic nuclei. The possibilities are still so numerous for significant advances away from stability that strong programs at the new heavy-ion machines can very successfully develop.

At GANIL, a He jet system will be used for this purpose and an on-line mass spectromete. 's being installed by the group from Laboratoire René Bernas in Orsay to study neutron rich nuclei with the same method. In source developments are expected to allow the study of halogen, Indium and Gallium isotopes ${ }^{\circ}$ ).

The process of projectile fragmentation, as opposed to the target fragmentation described above, was identified and used to observe new neutron-rich isotopes at heavy ion incident energies well above $100 \mathrm{MeV} / \mathrm{uma}^{21}$ ). It is well descritged in the participant-spectator model. In particular the width of the fragment mass distribution can be accounted for by the Ferni motion (see e.g. ref ${ }^{22}$ ). From recent compilations it seems that such a clearcut description is not valid if the 


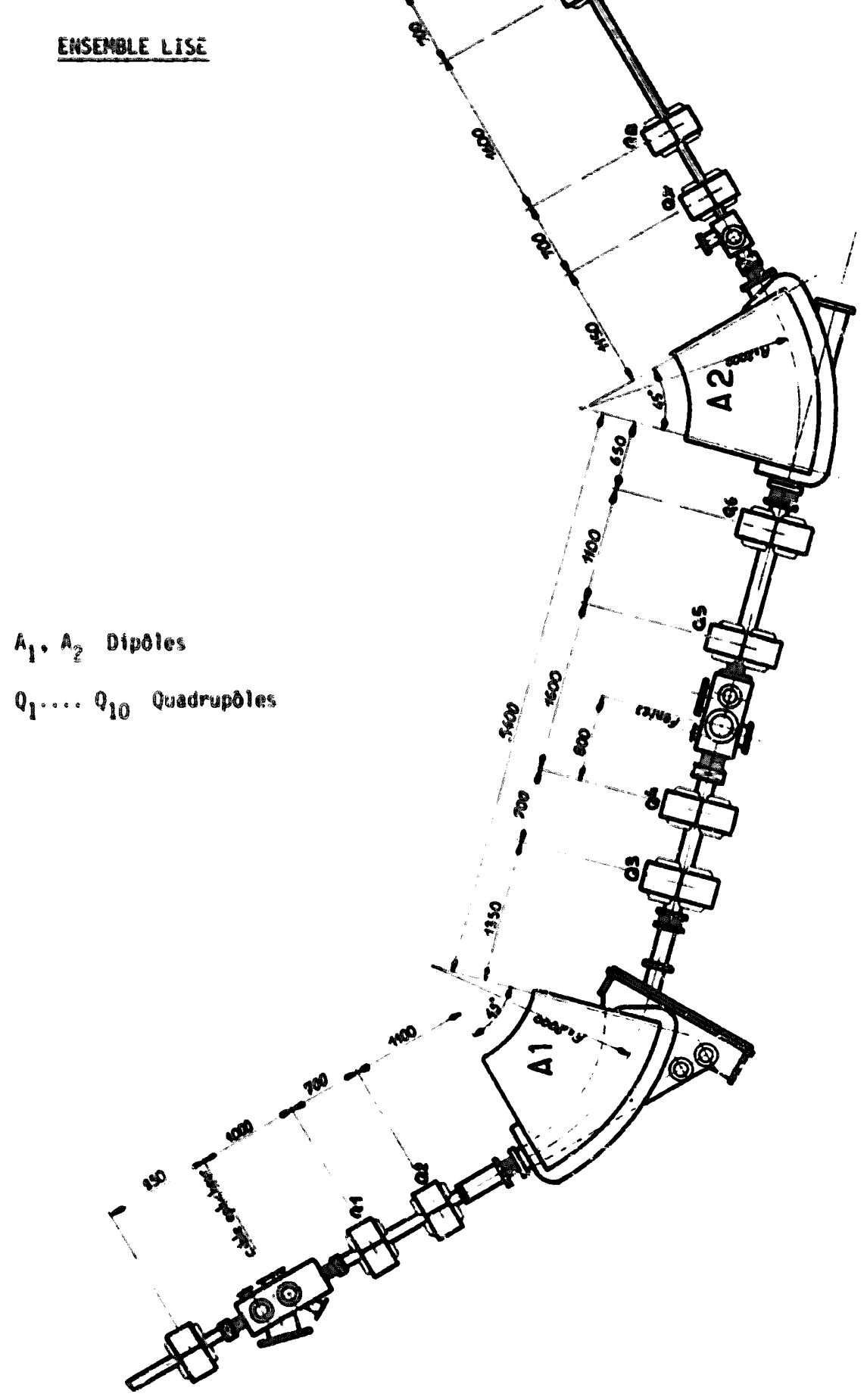

Fig. 4 The magnetic analysis of ions provided by the LISE beam line at GANIL (taken from ref ${ }^{24}$ ). 
incident energy does not reach $100 \mathrm{MeV}$ per nucleon ${ }^{19}$ ). Yet already with $45 \mathrm{MeV}$ per nucleon Argon beams, a large part of the cross section goes into a process where fragments somewhat lighter than the projectile are emitted in the forward direction with velocities close to the one of the beam ${ }^{23}$ ). Even if the width of the mass distribution does not follow the simple rule observed at higher energy, such a process is rather similar to projectile fragmentation. In any case it provides a very abundant source of nuclei moving within a rather narrow window in angle and velocity. Since the GANIL intensities are several orders of magnitude larger than those of higher energy heavy ion accelerators, a major breakthrough in extending the field of known isotopes towards the neutron drip line becomes possible $\left.\mathrm{e}^{26}\right)$.

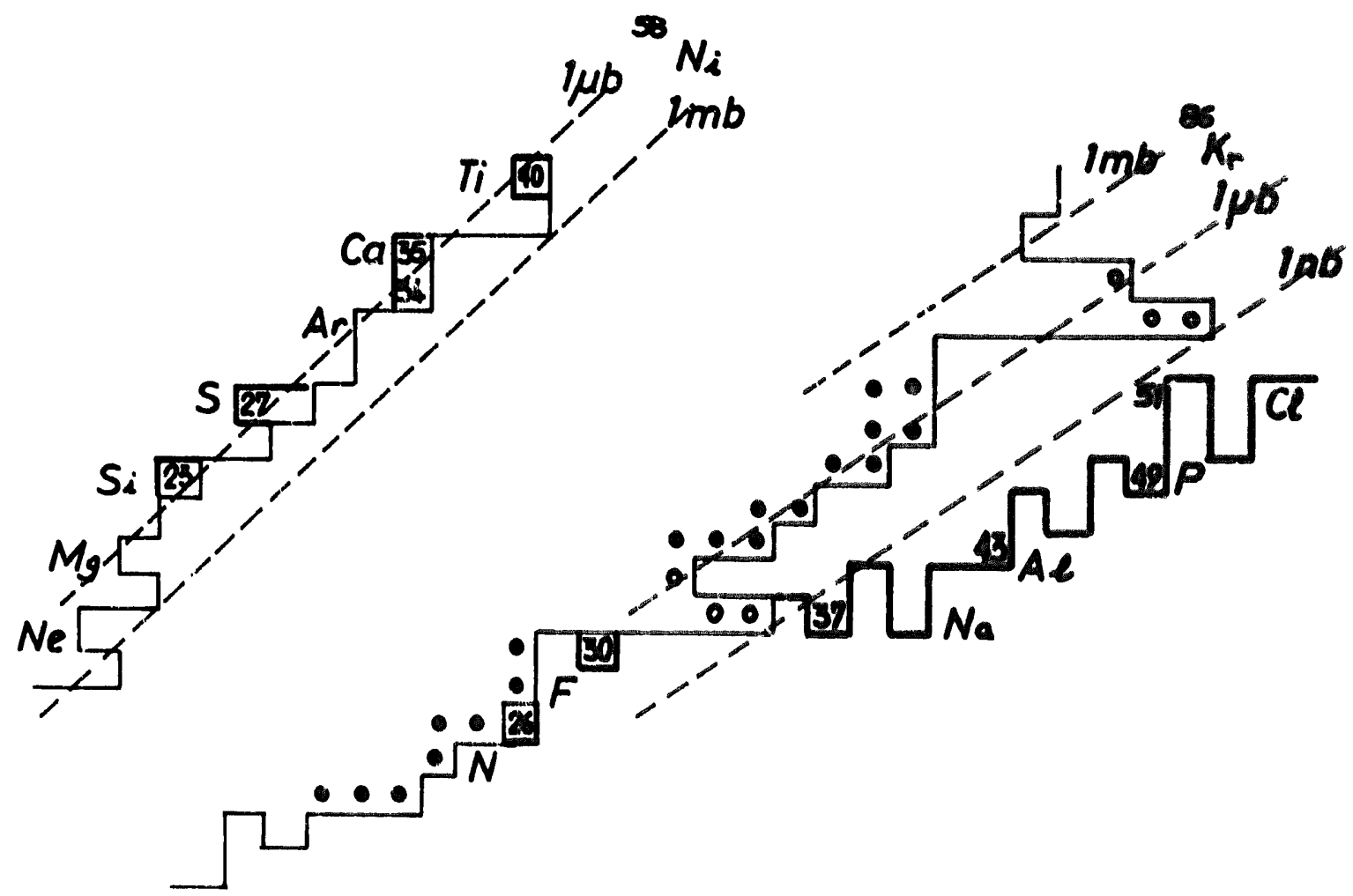

Fig. 5 A map of light isotopes indicating the new isotopes which could be reached by projectile fragmentation with the LISE facility at GANIL. Thick lines mark the predicted limit of stability of the isotopes for proton or neutron emission, thin lines the limits of known nucleides, open circles those recently discovered by projectile fragmentation at the Bevalac ${ }^{21}$ ), fill circles those studied with the Orsay on-line mass spectrometer $\left.{ }^{28}\right)$. The discontinuous line, taken from $\operatorname{ref}^{27}$, shows the anticipated cross section at GANIL energies for projectile fragmentation of $56 \mathrm{Ni}$ or $86 \mathrm{kr}$.

A faci: + $v$ is under compietion at GANIL, to be available by the end of 1983, which will allow the separation and identification of exotic nuclei produced by fragmentation. It is named LISE (Ligne d'Ions Super Epluchés, or super stripped ion line) and was originally designed to provide H-like or he-like heavy a toms for atomic studies such as beam foil spectroscopy. However it is also an excellent tool for the separation of rare reaction products $\left.{ }^{24}\right)$. It consists of two magnetic analyzers (fig. 4). The first one separates the reaction products according to their magnetic rigidity. For fragmentation products, most of which have the 
beam velocity, the $B_{0}$ value is proportional to $A / Z$. The second dipcle corrects for the dispersion of the first one. The system is then achromatic. In the simplest experiments the astered parameters of the ion will be its magnetic rigidity with a position-sensitive detector after the first dipoie, its time of flight, its energy loss $A E$ and ts energy. An unambiguous identification in $Z$ and $A$ will. result. The systentics of projectiie fragmentation cross section lead to the predictions given frifig. 5 for neutron-rich and neutron-deficient isotopes in the sd shell since the planned intensities of the new GANIL beam should allow around one even: per hour per nb cross section, many new isotopes should be identified. Furthernore techniques similar to the one used at the Bevalac to measure the ${ }^{22} \mathrm{Al}$ half $1 \mathrm{fe}^{2} \mathrm{y}$ wll allow the study of the radioactive decay of the identified isotope.

It seems that indeed the study of exotic nuclei has found a powerful new tool wh the occurrence of high energy heavy ion beams with intensities around $10^{\text {: }}$ particies/s. The ingenuity of physicists will be challenged by the need not only of observing many new isotopes but also collecting quantitative spectroscopic information about them.

\section{References}

1) Proc. of the 4 th Int. Conf. on Nuc 2 i far from stability (Helsingor 1981) CERN 81-09

$\left.{ }^{2}\right)$ A.G. Artukh et a1. Nucl. Phys. A176 (1971) 234

$\left.{ }^{3}\right)$ G. Muinzenberg et al. Zeitschr. Phys. A309 (1982) 89

") W. Benenson et al., Phys. Rev. C13 (1976) 1479

5) W. Benensois et al., Pruc. of the 3rd Int. Conf. on Nuclei far from stability (Cargèse 1976) CERN 76-13 p.235

$\left.{ }^{6}\right)$ R. Siemssen, invited paper at this Conference.

7) M. Bernas et al., Phys. Lett. 113 B (1982) 279

B) F. Naulin et al., Topicai Conf. on medium-light nuclei (Firenze 1977) Editrice Compositori ed. (Bologna, 1978) p.504

$\left.{ }^{9}\right)$ J.A. Nolen et al. Phys. Lett. B71 (1977) 314

${ }^{10}$ ) F. Naulin et al. J. de Phys. Lettres, 41 (1980) L79

11) G.D. Putt et al., Nucl. Phys. A., in press

12) F. Naul in et a1., Phys. Rev. C25 (1982) 1074

${ }^{23}$ ) L.K. Fifield, private communication

14) F. Naulin, private communication, and Ph D Thesis (Orsay, 1983) in preparation

15) D.M. Brink, Phys. Lett. $40 \mathrm{~B}$ (1972) 37

${ }^{16)}$ F. Naul in et al. J. de Phys. Lettres $\underline{43}$ (1982) L29

17) A.M. Poskanzer et al. Phys. Rev. C3 (1971) 882

R. Silberberg and C.H. Tsao, NRL Report 7593

${ }^{18)}$ M de Saint-Simon et al., Phys. Rev. C26 (1982) 2447

19) D.K. Scott, invited paper at this conference

20) A.C. Mueller and M. de Saint-Simon, private communication

$21)$ P.J. Symons et al. Phys. Rev. Lett. 42 (1979) 40 
1. Legrain, Proc. Int. Conf. on Selected Aspects of Heavy Ion Physics ISAclay 1982). North Holland pub1. p.219 c

4. Galln, 0. Guerreau et al., private communication

1. Ante and C. Signarbieux, GANIL report RA/NJ 278 (1982)

14. T. Symons, in ref $^{2}$ ), p.668

25) The possibility of using secondary beams to produce exotic nuclei which has itportant possibilities, is discussed by J.P. Dufour, A. Fleury and d. P. Bimbot, Phys. Rev. C23 (1981) 801. 